\title{
Robustness - Practice Report: Make Forming Processes more Stable Kai Kittner ${ }^{1, a^{*}}$ and Bernd Stimper ${ }^{1, b}$
}

\author{
${ }^{1}$ Eberspächer Exhaust Technology Wilsdruff GmbH, Global Manufacturing Engineering \\ Commercial Vehicle, Hühndorfer Höhe 33, 01723 Wilsdruff, Germany
}

akai.kittner@eberspaecher.com, bbernd.stimper@eberspaecher.com

Keywords: robust processes, statistical simulation, numerical simulation, industry 4.0

\begin{abstract}
In the last years, the development of sheet metal forming technologies show many different tendencies such as lightweight constructions with high strength materials, thinner materials or stiffer geometries. In the same period of time the process planning for stamping processes remain in the same way, although numerical algorithm and hardware improved continuously. This implies, as components, processes and materials got more complex, the planning process still stays in the same technical evolution stage as years before. At present it is mostly a deterministic kind of planning. And in this way, potentials and possibilities are still unused. In the current discussion concerning industry 4.0 new potentials seem to show new chances. A lot of data and technologies are already available to improve production and forming processes. The challenge is to utilize this data. The key to improve the processes based on this data is the statistical simulation, especially in the sheet metal forming industry. Although the method of statistical simulation and robustness engineering do not reached a wide base, several authors investigated own processes with this method. [1-6].

The Eberspächer Company as a customer of sheet metal parts has an immense interest in high quality parts. Such parts are an important step to reduce cost in the own manufacturing. The company assesses the statistical methods as a probate way to solve the problems around springback and geometric accuracy on components. This paper presents activities to integrate stochastic considerations into the planning processes at Eberspächer. And its content contributes the discussion around robustness processes, stochastic simulation and in this way industry 4.0 as one fundamental base for manufacturing.
\end{abstract}

\section{Introduction}

The Eberspächer Company is one the world leading supplier for EU6 exhaust systems. The exhaust technology section of the company is divided into a passenger cars unit and the business unit (BU) commercial vehicles. The plant in Wilfsdruff is assigned to the BU commercial vehicles and delivers after treatment systems (ATS) to several OEMs, such as Volvo or Mercedes, figure 1.
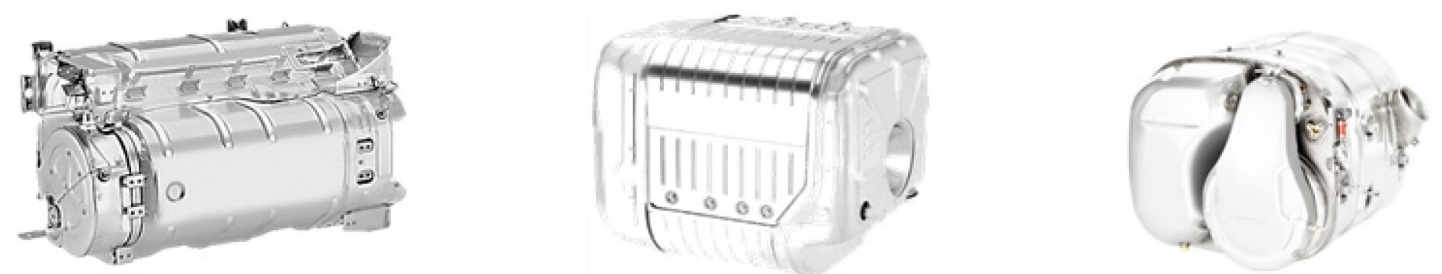

Fig. 1. Examples of ATS-systems for trucks build up in Wilsdruff Eberspächer plant [7].

The major part of ATS are different kind of catalysts depending on European exhaust standards (e.g. EU5, EU6). The catalysts are:

- DOC: Desoxidation catalysm

- DPF: Diesel particle filter

- SCR: Selective catalysm reaction (Ad blue, Urea injection) 
Furthermore the ATS-systems mainly consist of sheet metal forming components for structural reasons, gas guidance and thermal isolation. The majority of these components are built up in a deep drawing process. In the plant the single components are assembled to the complete ATS-system. The core technology for assembling is the welding process, in detail the MAGwelding process. For positioning and fixing in each operation welding fixtures are used. Figure 2 depicts an example of an ATS which was assembled in Eberspächer plant Wilsdruff.

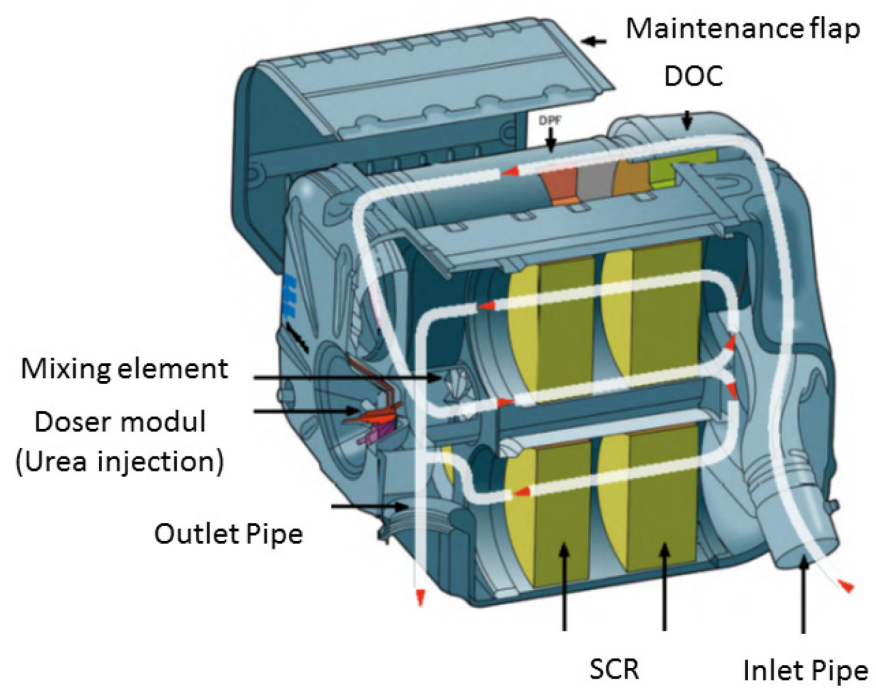

Fig. 2. More detailed visualization of ATS [7].

A main challenge is to meet the quality requirements of the sheet metal components. Therefore, the dimensional accuracy is not sufficient and often outside the defined tolerance bands. This leads to problems during the welding process, because the gaps between different components fluctuate in a wide range. Following failures can be insufficient connection between the components and cracks in the welding seams. The problem around springback is getting more complicated with the statistical behaviour of the forming process. That means, the forming process is not static, not deterministic; the process fluctuates due to its input parameter, like mechanical properties of material or friction, fig. 3.

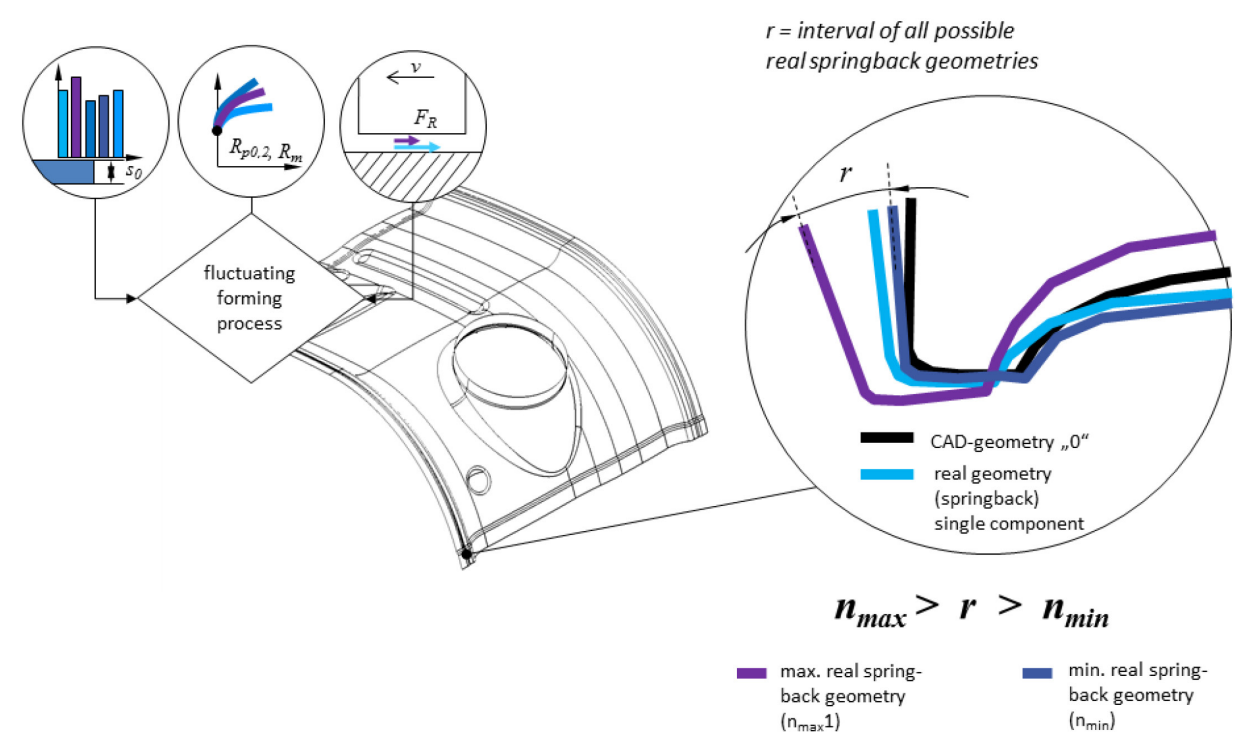

Fig. 3. Schematic of the initial problem "springback".

Besides the initial problem, springback behaviour/ and fluctuating welding conditions, there are some issues more which make the situation more complex. Along the process chain there are the different departments of a company and different data are generated. Main involved departments are development (engineering), quality and production/ manufacturing engineering (ME). In the 
planning stage the springback is computable by the numerical simulation and this is the base for tool compensation. State of the art is a deterministic simulation. In the approval stage, the quality department measures and evaluates later the quality of real components. In general, every single measurement report for every single part is checked. Sometimes little statistical analysis is applied. Based on this data, then the ME- engineers adapt fixtures in the production, if any problems occur during initial implementing the welding. So to deal with the springback behavior is a topic of daily work in many departments. Unfortunately, some of them generate data about that but only for individual documentation. This implies, the level of horizontal data integration can be improved and at the end, the existing data are not consequently used for an improved planning. Figure 4

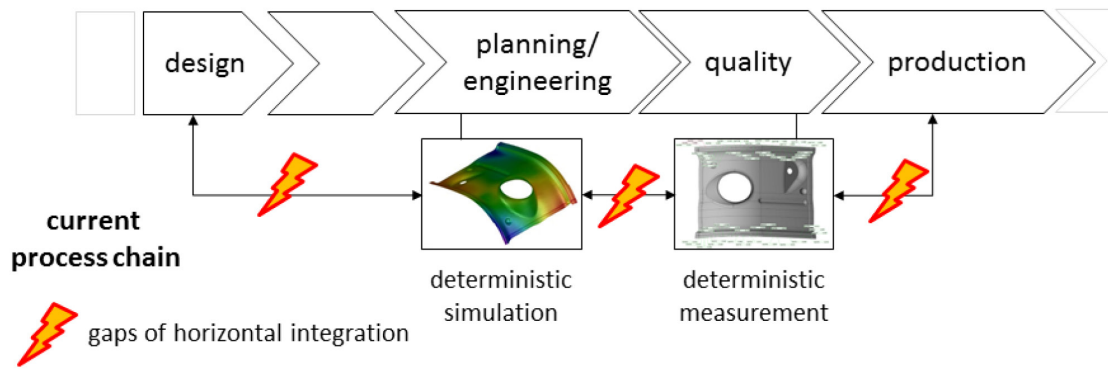

Fig. 4. Schematic of some departments along the process chain and horizontal data integration of components.

The problem is to merge the information and suitable visualization of data. The target of internal activities is to find an approach for an improved process planning and better part quality and the focus of this presented paper. Key word of this work is robustness. The aim is to control the statistical behaviour of the forming process and in this way the springback behaviour. At first, a little view is given to improve the part quality and handle problems regarding springback and geometric accuracy. At second, a concept for future planning is sketched.

There are some possibilities to compensate insufficient component quality (Fig. 5). Seldomy used possibility is the design adaption. In this case some geometric features, like little reinforcement beads, are inserted in the design of the component in order to improve stiffness behavior resulting in a lower springback. Otherwise, it is possible to order material with a smaller range of mechanic strength specification than the DIN standard. Alternative possibilities are recalibration of welding fixture and welding process. An expensive way to improve the quality is the iterative (trial and error) way to adapt the tool with changes in active surfaces, radii or process parameters like blankholder force.

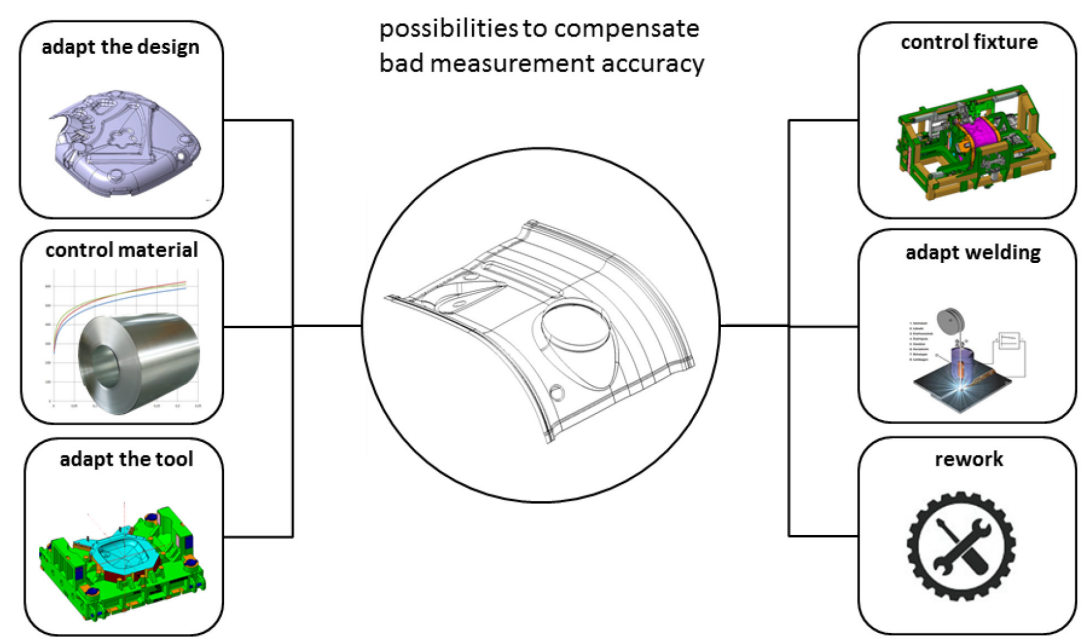

Fig. 5. Possible actions to compensate insufficient component measurement. 
A comparison gives an overview on planned tool costs and additional costs, caused in compensating actions, see previously paragraph and figure. Here (only as percentage presented) it can be seen, there is a gap between planned tool costs and the real costs. Fig. 6 gives an overview about this situation. The base is the planned costs and is represented with $100 \%$ of costs. Regarding the experience in projects, it is possible to collect and extrapolate additional costs over the whole life cycle of a component. Additional costs result from:

- additional project management effort for coordination, supply chain management, engineering, suppliers and customers

- process internal rework

- scrap

- tool and fixture changes.

In particular cases can these leads to additional costs up to 40 percent for components and/or tools Average 20 percent of additional costs can avoided by an improved process planning. In comparison to the increased planning effort there is a gap of 15 percent to reduce additional costs. But it has be the goal to stay within the planned cost.

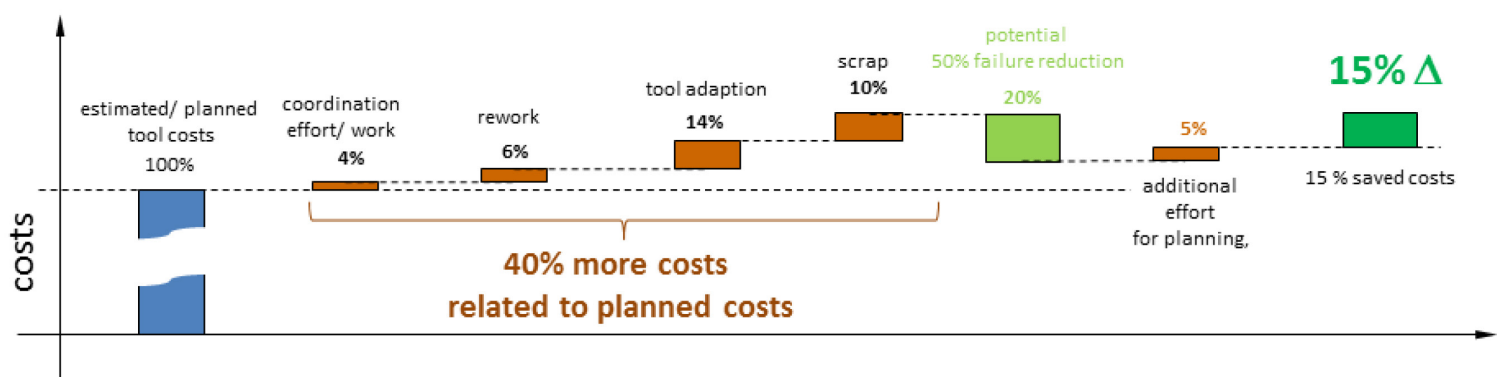

Fig. 6. Expertise about planned cost (blue) an additional costs (i.e. rework, brown color), furthermore the potential of saved costs for a better planning process.

\section{Examinations}

The internal Eberspächer projects have started in cooperation with German VDI association, universities and universities of applied science. The work is mainly divided in three major topics

- quality assessment of welding seams and rating of welding failures

- investigation of measurement reports and further analysis

- numerical simulation of forming processes as statistical processes and analysis of springback behaviour (statistical) an comparison to practice statistical behaviour

For reasons of confidentiality it is only possible to show results in excerpts. A decisive part of this investigation is robustness/ robust processes. With robustness and thus statistical considerations a better understanding of forming process and their influences can be achieved. Outgoing from the first main topic, the difference between out of tolerance and good welding quality is in consequence depicted in Fig. 7.
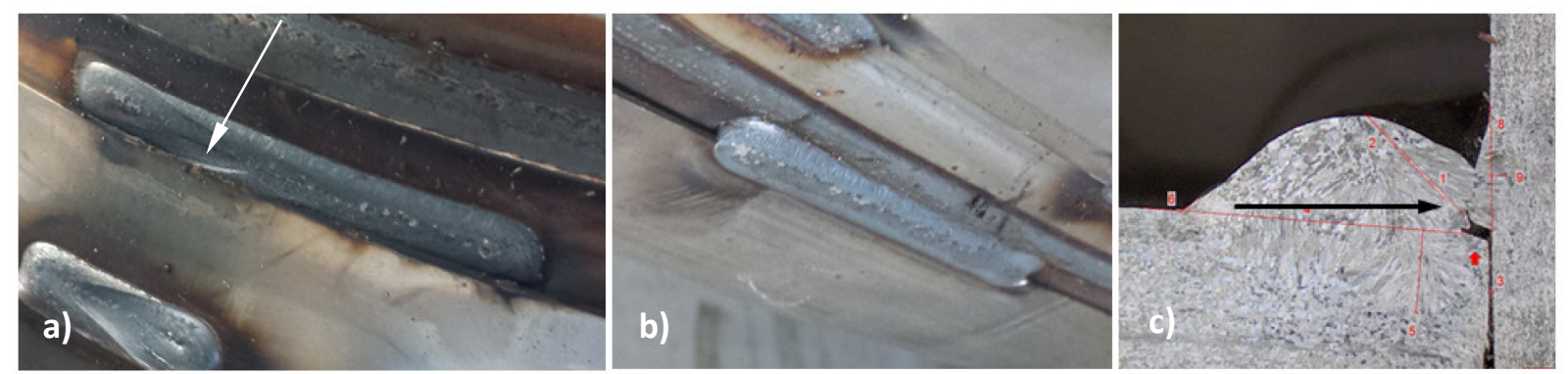

Fig. 7. a) Bad welding quality; insufficient welding seam, bad connection to overlapping sheet b) Good welding quality, welding seam without any abnormalities, c) Bad connection of components (crack). 
The chosen example for investigations is an outer shell component. This kind of part provides the biggest complexity in assembling/ welding. This is caused through the size of the components itself and in the more flat than structural character in the part geometry. So the components do not have a sufficient own stiffness and leads to the already mentioned springback behaviour.

The second investigation step was the analysis of measurement reports. The analysis of the reports showed future requirement on these reports. The problem on the actual workflow regarding the reports is explained in the following. In a standard evaluation process for a component 10 parts are necessary. The supplier delivers 10 several measurement reports for each part. In the approval stage the reports are checked in all specifications, especially keep of tolerance bands in welding areas. It is quite difficult to check all measurements and keep the values in mind and compare them. Especially, if there are any problems with the accuracy, it is barely possible to realize the complex measurement situation over all part und derive the correct actions for an improvement. The analysis of each single measurement report for each component is common procedure in quality assessments in most of known companies regarding sheet metal forming.

Another, preferred way of analysis is conducting a statistical analysis of chosen parts. This analysis gives a better understanding over the measurement of all parts. Fig. 8 shows the measurement analysis. Statistical results are average value $(\mu)$, variation $(\Delta$, difference between highest and lowest value) and standard deviation $(\sigma)$.

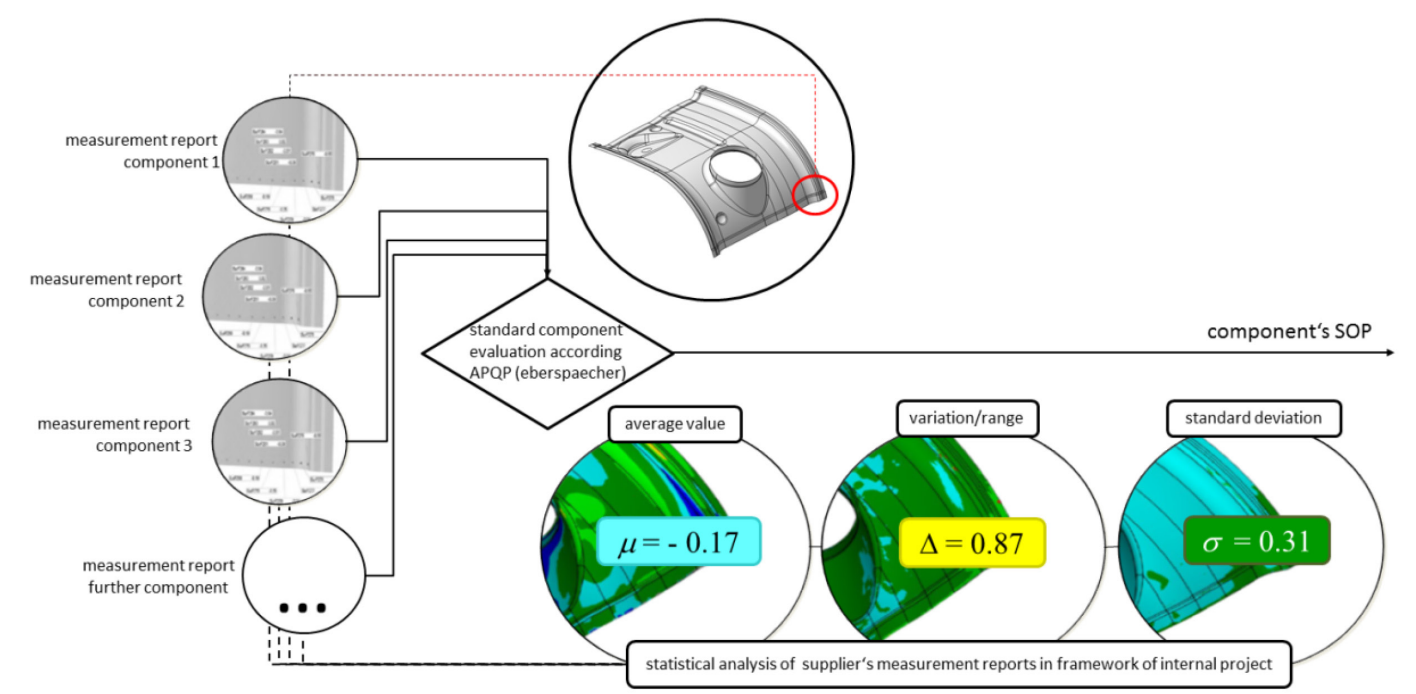

Fig. 8. Statistical visualization of all 10 measured parts, average value $(\mu[\mathrm{mm}])$, variation (difference of highest and lowest value, $\Delta[\mathrm{mm}]$ ) and standard deviation $(\sigma)$.

In the example in fig. 8 and 9 , it can be seen for the considered area the average value of all parts is inside the tolerance band $(+/-0.4 \mathrm{~mm})$. Basically it is a preferable result. But the variation and standard deviation are rather high. That means, the process is inconstant with fluctuation. And in this case an adaption of tool is not useful. Because the tool adaption influences the average value positively in this way, but it can be expected that the variation and the standard deviation stay in the same range. Thus, a high percentage of components furthermore cannot fulfill the requirement.

Figure 10 shows the problem around this component (all of these components) in detail. The outer corners have a high deviation in the average value and variation. Similar to the statement in the paragraph before, the situation is very complex and only an adaption of tool is not useful. An adaption needs fundamental changes in drawing and forming stages. If the tool already exists, big changes are often not possible or only with a high effort. To solve this kind of measurement situation multilevel actions are necessary. On the one hand, the fixture has to compensate the measurement deviation. This requires an adjustable fixture with high clamp force. On the other hand, the tolerance chain computation has to be adapted with the focus to allow a bigger tolerance band for the outer shell component. For this complex situation, the requirement for a better a statistical planning/ simulation is obvious - control the process with robustness engineering. 


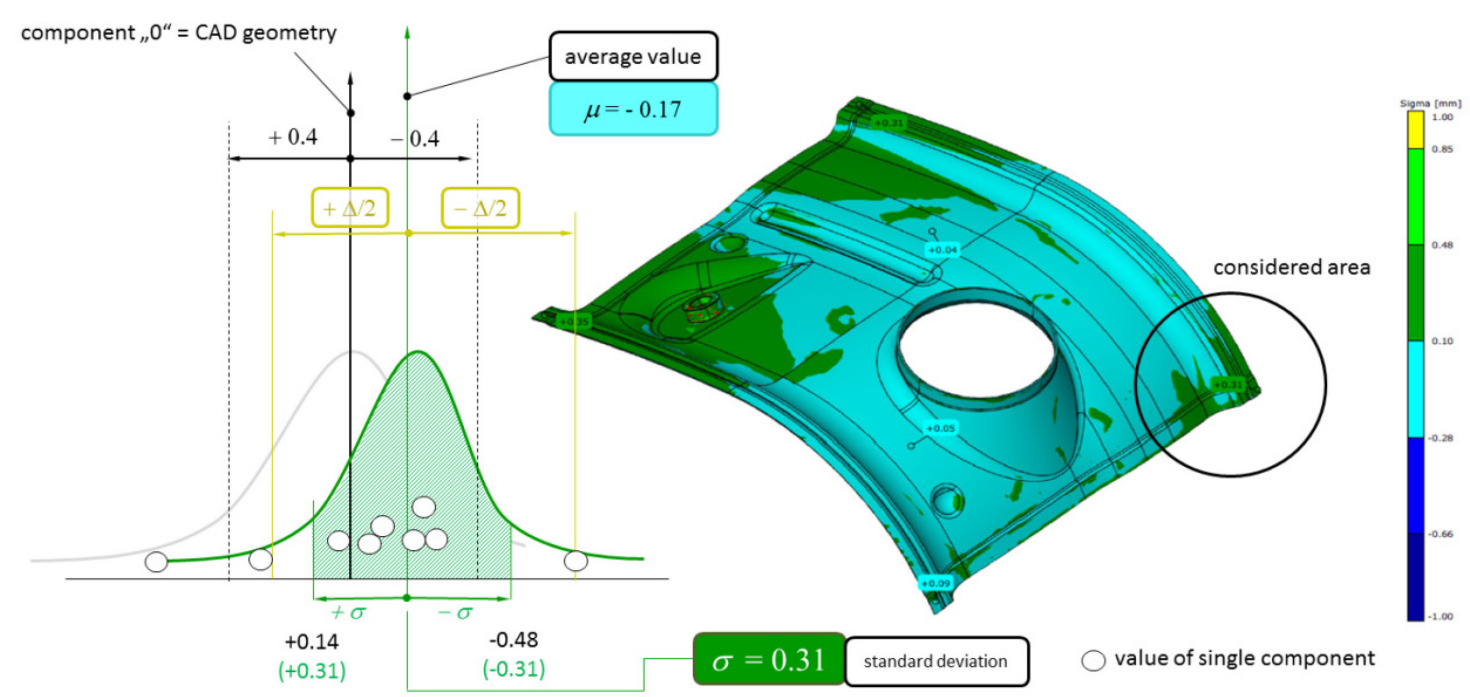

Fig. 9. Analysis of considered area; in this area a high percentage number of components is outside the tolerance band, the components cannot fulfill the requirement in geometry accuracy, additionally the process fluctuates.

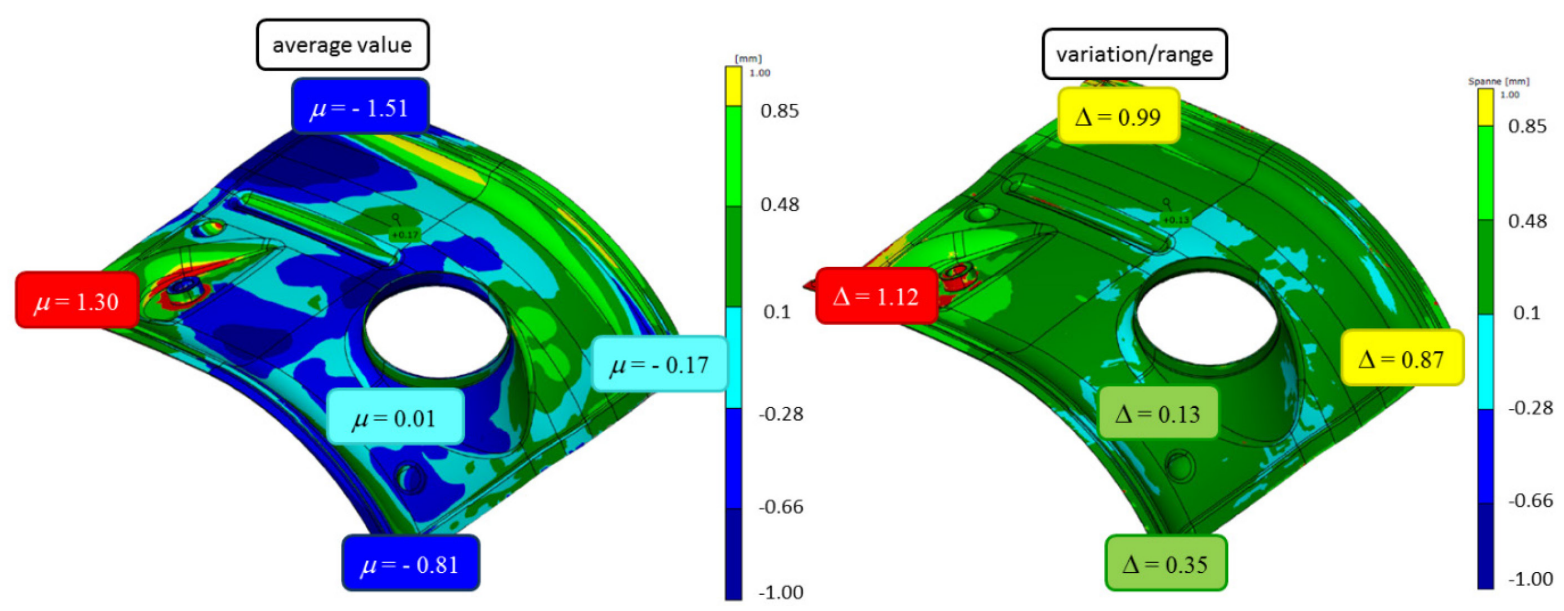

Fig. 10. Overview about the whole part and the quality, left hand side: average value; right hand side: variation.

In the last examination step the springback behavior for different components, also the example outer shell, were analyzed. For the simulation the Autoform software, version R5.1, with the sigma feature was used. The Sigma- add-on allows the computation of statistical behavior of a forming process. The database consists of yield data from Eberspächer experiments and the active surfaces from tool maker. The nominal material is a ferritic stainless steel, 1.4509, with a sheet thickness of $1.2 \mathrm{~mm}$. The component is built up in 6 tool stages. The last and sixth computation step is a springback computation. In the practice, the part is measured in a measuring fixture with standard industrial clamps around the outline of the part. In the simulation, a similar clamping concept was used. So the simulation results have the same base for comparison to measured parts from the supplier.

The design space variables were yield strength and tensile strength in variation according to German DIN standard, friction coefficient for Coloumb's friction between 0.1 and 0.15 , sheet thickness of $1.15 \mathrm{~mm}$ up to $1.25 \mathrm{~mm}$ and a variation of initial blank position before first forming step. The design plan was created automatically by the Autoform software according to a Latin hypercube standard. 

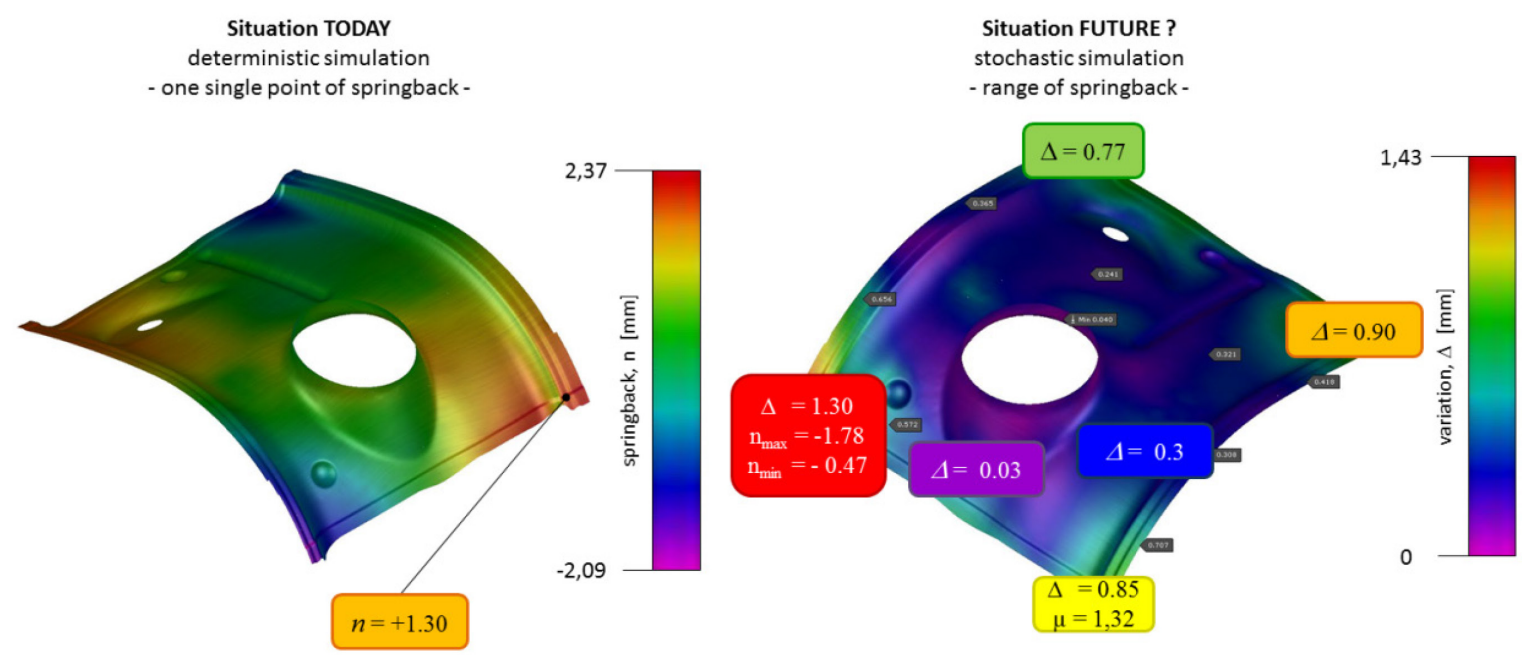

Fig. 11. Overview about the whole part and the quality, left hand side: average value; right hand side: variation.

Figure 11 shows the difference between the current "state of the Art" in springback computation (deterministic simulation) and the possibility to compute the forming process as a statistical process. In the computation and analysis the visualization is similar to the analysis of the measurement reports. The simulation computes average values, variation, standard deviation and a lot more results. The result of computation shows the same quality of springback. That means, the components have, caused in its geometry and the forming process, a twist in itself. All four corners have high springback behaviour and a high variation of springback values. Differences exist between the correct values of springback between simulation and practice. But the result offers already the potential for future process planning. The simulation is able to compute the springback behaviour and the fluctuating values. With the knowledge of fluctuating results it is possible to react in an early stage of planning and make the following processes robust against the changing quality (springback behaviour).

\section{Results and Conclusion}

The examinations within the projects showed:

- along the process chain a lot of data is generated

- a fluctuating component quality is the reason for fluctuating quality in welding processes and lead to high compensating actions

- geometrical deviations are known, but the comprehensive "picture" of the fluctuating, statistical process behaviour is not clear

- a statistical behaviour of forming process and in order the range of springback is computable, but it is not regularly used

- methods and knowledge to make a robust process design are available, but not used.

At this point of investigation, it is clear, without a wider understanding of robustness and statistical behaviour there is no improvement of components and furthermore for the welding quality possible. The base for an improvement is already available. It is necessary to adapt the knowledge into the process chain, especially in the planning stage. The integration of the concept occurs in two steps. The deterministic measurement and the deterministic simulation go over to statistical ones. Furthermore, a reverse engineering analysis over several parts will be conducted. It is a kind of calibration of the numerical simulation to reduce difference between simulation and practice. In the second step, the statistical simulation will be integrated directly in the planning process. Based on the results of statistical simulation the design department can directly adapt the part (reinforcement beads, bigger tolerance bands), the manufacturing engineering department develops fixtures with adequate adjustments and clamping and the tool maker can adapt the method more robustly. Fig 12 shows the concept. So Eberspächer decided to move on according robustness and an implementation phase of two years is estimated. 


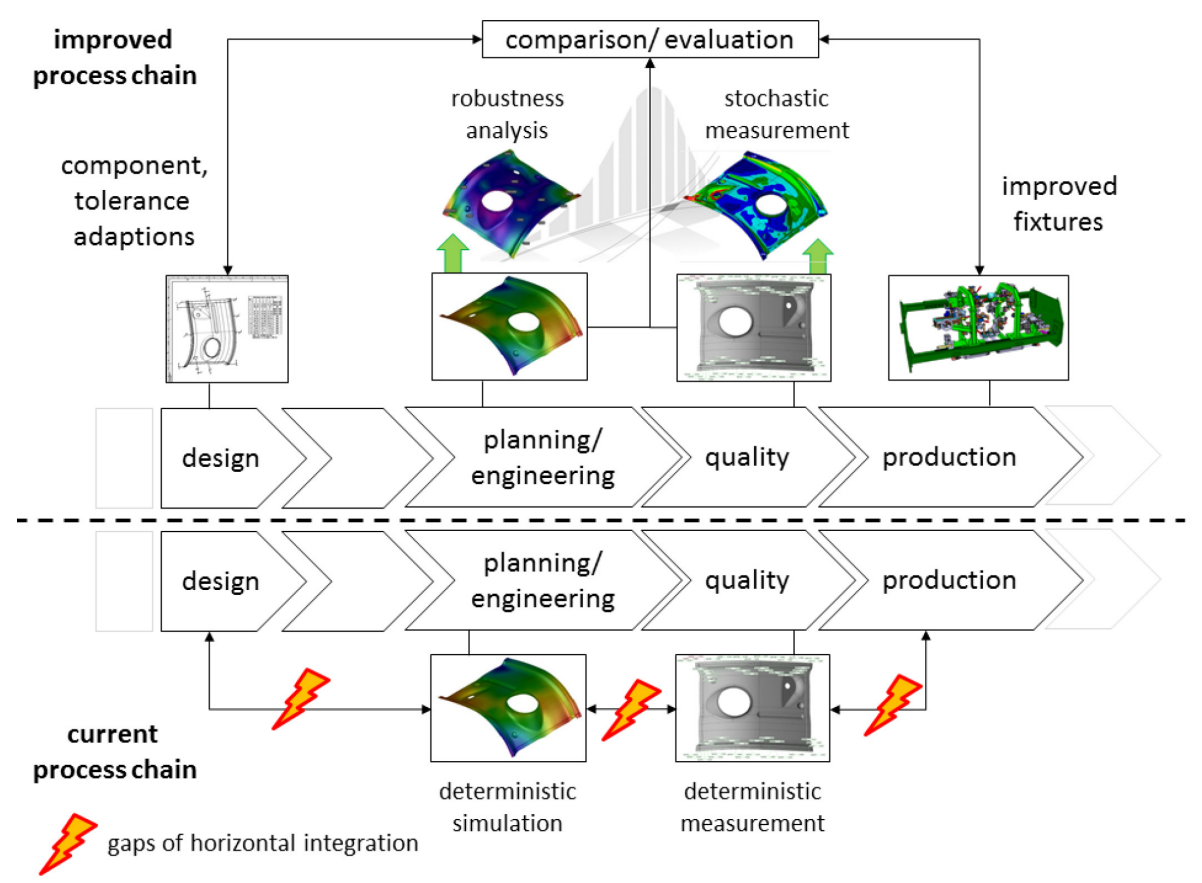

Fig. 12. Integration of robustness planning/ simulation in the planning concept.

\section{References}

[1] E. Atzema, M. Abspoel, P. Kömmelt, et al., Towards robust simulations in sheet metal forming, Int. J. Mater. Form. 2 (2009) 351.

[2] K. Kittner, S. Kunath, S. Wolff, et al., About the requirement of robustness engineering in the future of forming processes. 5th International Conference on Accuracy in Forming Technology ICAFT 2015 and 22nd Saxon Conference on Forming Technology SFU 2015 - Conf. Proc. ISBN: 978-3-95735-029-9.

[3] Wolff, S.: Robustness Analysis of Metal Forming Simulation - State of the Art in Pratice. 5th International Conference on Accuracy in Forming Technology ICAFT 2015 and 22nd Saxon Conference on Forming Technology SFU 2015 - Conf. Proc. ISBN: 978-3-95735-029-9.

[4] Schmidt-Jürgensen, R.: Untersuchung der Simulation rückfederungsbedingter Formabweichungen beim Tiefziehen. Dissertation Universität Hannover, Hannover, Deutschland, 2003.

[5] H. Wiebengaa, E.H. Atzemab, Y.G. Anb, H. Vegterb, A.H. van den Boogaard: Effect of material scatter on the plastic behavior and stretchability insheet metal forming. Journal of Materials Processing Technology 214 (2014) 238- 252.

[6] Benny Endelt, Søren Tommerup, Joachim Danckert: A novel feedback control system Controlling the material flow in deep drawing using distributed blank-holder force. Journal of Materials Processing Technology 213 (2013) 36-50.

[7] Eberspächer company, internal, 2017. 